EPJ Web of Conferences 32, 02001 (2012)

DOI: $10.1051 /$ epjconf/20123202001

(C) Owned by the authors, published by EDP Sciences, 2012

\title{
Summary of Papers on ECRH Experiments
}

\author{
S. Kubo ${ }^{1, a}$ \\ National Institute for Fusion Science, Toki 509-5292, Japan
}

Abstract. The presentations on ECRH experiments presented in EC-17 are summarized.

\section{Real time control, feedback control}

One of the hot topics of ECRH experiment discussed in this workshop was real time control or feed back control fully utilizing local controllability and fast time response of the ECRH system. The real time system for MHD activity control in the FTU tokamak is presented by C. Sozzi, where the several hardware and control argolism for the feedback control of MHD activities (sawtooth, NTM) are implemented and initial test was successfully executed. Related series of papers on FTU are presented by E. Alessi, C. Galperti and A. Moro. ELM control or pacing as well as the suppression of the MHD instabilities is also a hot topic of ECRH experiment and outstanding experimental results in TCV are presented by F. Ferici and M. Lauret. Utilizing newly installed real-time antenna control system with flexible digital real time control system, sawtooth pacing, ELM pacing, NTM suppression are demonstrated. J. Lohr also reported a fine control scheme developed and executed in DIII-D using the plasma control system (PCS) and field program gate array (FPGA) witch are connected also to ECRH system allowing fast control of the injection angle and gyrotron output power. These capabilities are utilized to control plasma instabilities and secure operation of the gyrotrons as well. New fast steerable launcher and "NTM feedback suite" (equilibrium, density profile, ray tracing, correlation analysis, mirror control) are implemented in ASDEX U are also presented in the talk of J. Stober. Feedforward test of the system were already done and waiting for the feedback control demonstration. ECH and ECCD effects on NTMs stabilization by ECRH is also investigated for JT-60SA tokamak and presented by C. Sozzi.

\section{ECRH system upgrades and demonstration of its potential}

In the presentations of the application of ECRH on the DIII-D Tokamak, R. Prater thoroughly reviewed potential of ECRH in the investigation of the tokamak operation and indicated the upgrading scenario of the ECRH system in the DIII-D. Topics included are the electron heating effects related to the study of plasma rotation and $\mathrm{H}$-mode threshold power, $\mathrm{T}_{\mathrm{e}}$ profile control for validation of the NBCD model or RWM control, modeling ITER startup and rampdown, modifying the temperature gradient for studying the transition, trapped electron mode, and QH-mode. ECCD was applied to drive currents in the plasma in ITER demonstration steady-state discharges for radially distributed current drive in support of fully noninductive operation at high beta, control of neoclassical tearing modes for experiments on resistive wall modes in low rotation, high beta discharges, for neoclassical tearing

\footnotetext{
a e-mail: kubo@lhd.nifs.ac.jp
} 
mode control using oblique electron cyclotron emission as a diagnostic to determine robustly the optimum location of the ECCD, studies of the validation of resistive MHD models and development of model-based current profile control. In accordance to the increasingly important role of the ECRH, it is reported that the ECRH system is proposed to be upgrade to total injection power up to $12 \mathrm{MW}$. In ASDEX U, J. Stober reported undergoing upgrade of multi frequency system $(140 / 105 \mathrm{GHz})$ and injected power reached 3.9 MW at $140 \mathrm{GHz}$ and $2.1 \mathrm{MW}$ at $105 \mathrm{GHz}$. Such enhanced ECRH power was used for several physics studies such as H-mode optimization. In relation to the system upgrades, monitoring the stray radiation in the vacuum vessel is also an important issue and M. Schubert discussed the Sniffer probes and large area bolometer detectors. J. H. Jeong reported the successful commissioning of $170 \mathrm{GHz}, 1 \mathrm{MW}$ ECH\& CD system in KSTAR. High power long pulse injection experiments are expected to start soon.

\section{Detailed Physics Study}

\section{Confinement}

M. van Berkel discussed the determination of localized heat transport in fusion plasmas using ECRH and ECE. They developed developed a new identification method to estimate the different components of the one dimensional radial heat transport as function of the radius and the method was tested using finite difference simulations in the presence of additive noise and it was shown that it is possible to estimate the local diffusion coefficient, damping term and convection velocity in slab geometry with uncertainty.

\section{High energy electrons}

$\mathrm{X}$. Ding gave an invited talk on energetic particle physics with high power ECRH on HL-2A. The high power ECRH is used to study high energetic particle physics which becomes important in f plasmas with high power heated or fusion burning plasma. The electron beta induced Alfven (e-BAE) mode is excited in HL-2A under $600 \mathrm{~kW}, 68 \mathrm{GHz}$ ECRH. S. Kubo discussed the production of the high energy electrons and its effect on the ECRH in LHD. With much production of the high energy electrons, relativistically down shifted higher harmonic resonance appears in case of low field side injection and the power absorption there can alter the ECRH power deposition.

\section{Electron Bernstein Wave (EBW) Heating}

Only two paper related to heating proper experiment or system are presented from LHD and NSTX-U. $\mathrm{H}$. Igami reported the EBW heating results for slow O-X-B mode conversion scenario. Apparent but small heating effect is demonstrated on the over-dense plasma using $1 \mathrm{MW}, 77 \mathrm{GHz}$ power in LHD. Here, ECCD in LHD is also investigated from the change in the rotational transform and its effects on the transport. G. Taylor presented a conceptual design of the ECRH system on NSTX-U. Due to the enhancement of the toroidal field coil, $28 \mathrm{GHz} 1 \mathrm{MW}$ system is under consideration. The system is expected for an efficient breakdown, fundamental EBW heating and current drive for NSTX-U plasma equilibria. It should be noted that several EB-Emission measurement system in view of the EBW heating optimization are underway and presented in the ECE session.

\section{Plasma production/start-up}

For establishing the ITER start-up scenario, breakdown and current startup experiment have been done in several machines DIII-D, JT-60U, etc. New reports from T10, KSTAR and FTU are presented in this meeting. A. Borshchegovskiy reported Optimization of ECR-breakdown and plasma discharge 
formation on T-10 using X-mode second harmonic of ECRH. It is demonstrated that small plasma equilibrium toroidal current gives optimal initial plasma parameters. M. Joung reported the second harmonic $110 \mathrm{GHz}$ ECH-assisted startup in KSTAR. ECRH injection during the toroidal current rampup phase is effective but needs poloidal field control to keep the plasma equilibrium stable. G. Granucci reported the studies on absorption of EC waves in assisted startup experiment on FTU.The effect of oblique injection with the wall reflection gives efficient build up of the plasma. 